\title{
Photometry of two DQ white dwarfs. Search for spots ${ }^{\star}$
}

\author{
T. Vornanen and A. Berdyugin
}

\author{
Department of Physics and Astronomy, University of Turku, Vaisalantie 20, 21500 Piikkio, Finland \\ e-mail: tommi .vornanen@utu.fi
}

Received 21 March 2012 / Accepted 11 July 2012

\begin{abstract}
Aims. The intensity profiles of the $\mathrm{C}_{2}$ Swan bands in cool DQ white dwarfs cannot be adequately fitted with models that otherwise succesfully reproduce spectral features of the molecule $\mathrm{CH}$ in these stars. Initial modelling showed that a two-component atmosphere in the style of a spot might be able to solve the problem.

Methods. We photometrically observed the two cool DQ white dwarfs GJ1117 and EGGR78 to search for variability caused by stellar spots.

Results. We have not found any such variability, but we estimate the effects of hypothetical spots on lightcurves. We also estimate detection probabilities for spots in different configurations. Alternative explanations of the problem are needed and briefly discussed.
\end{abstract}

Key words. white dwarfs - starspots - stars: atmospheres

\section{Introduction}

Magnetism in white dwarfs (WDs) is usually studied through Zeeman splitting of atomic spectral lines. This method has proven to be very useful, and with the sensitivity added by spectropolarimetry it is very effective in detecting magnetic fields. Spectra of cool DQ WDs do not show any atomic lines, therefore an alternative method is required. Fortunately, the carbon molecules present in most of these stars are sensitive to magnetic fields and they can be studied using spectropolarimetry.

During the past several years we have used circular spectropolarimetry to observe known DQ WDs to look for polarization signals from $\mathrm{C}_{2}$ and $\mathrm{CH}$ molecules that can be found in their atmospheres. We have used two different telescopes, the Nordic Optical Telescope (NOT) on the Canary Islands and the ESO VLT (Cerro Paranal, Chile). So far, we have observed 12 objects and found one of them (GJ841B) to be magnetic (Vornanen et al. 2010). The rest of the stars do not show any polarization signal at the noise level of our observations, i.e. $0.5 \%$ in Stokes $V / I$ for the NOT observations and $0.2 \%$ for the VLT observations.

In Vornanen et al. (2010) we showed that the model presented in Berdyugina et al. $(2005,2007)$ works very well for the $\mathrm{CH}$ absorption bands in GJ841B and were also successful in modelling the $\mathrm{CH}$ and $\mathrm{C}_{2}$ blend at $430 \mathrm{~nm}$ visible in both $\mathrm{G} 99$ 37 and GJ841B. But while we modelled the intensity profiles of the purely $\mathrm{C}_{2}$ Swan bands, we were unable to achieve good fits. Problems with fitting Swan bands in DQ WDs have been reported before (for example, Dufour et al. 2005 have shown that the $\Delta v=+2$ bands are consistently too strong in their model fits). Our model shows the same problem (as seen in Fig. 1), but in addition to that the $\Delta v=0$ band appears too weak. Hints of a too weak $\Delta v=0$ band can also be seen in Dufour et al. (2005). These problems persisted for all non-magnetic DQ WDs in our survey, as long as we used a single temperature model.

\footnotetext{
* Photometric data is only available at the CDS via anonymous ftp to cdsarc.u-strasbg.fr (130.79.128.5) or via http://cdsarc.u-strasbg.fr/viz-bin/qcat?J/A+A/544/L6
}

We started searching for a solution to this problem by combining two models in the manner of a photosphere with a spot to obtain reasonable fits to the spectra. This led to somewhat strange results. The best-fitting models usually had a small spot with $T=8000-10000 \mathrm{~K}$ and a photosphere of $T=2000 \mathrm{~K}$. This would suggest a cool disk or an envelope of gas or dust around the WD with a hole in it through which the atmosphere would be seen. Although dust disks have been found around hot WDs and the central objects of planetary nebulae (Bilíková et al. 2011), this does not sound like a very probable scenario for our cool objects. Instead, a cool spot has been found before on a weakly (70 kG) magnetic WD (Brinkworth et al. 2005).

To exclude spots or envelopes with holes as the reason for the observed properties in these WDs, we started a photometric observing programme with a small remotely controlled telescope on the Canary Islands. Since WDs are rotating just like any other star, the movement of the spot on the visible hemisphere would cause some photometric variability. We report the results of our photometric observations here and discuss what we can deduce from them. At the end of the paper we discuss other possible reasons for troubles with modelling.

\section{Observations}

For our observations we used the $35 \mathrm{~cm}$ Schmidt-Cassegrain Celestron telescope attached to the side of the $60 \mathrm{~cm} \mathrm{KVA}$ (Kungliga Vetenskapsakademien) telescope in Observatorio del Roque de Los Muchachos (ORM) on the island of La Palma in the Canary Islands. The telescope is remotely operated by researchers at Tuorla Observatory of the University of Turku (Finland). The small telescope has filters for BVRI photometry and is equipped with an Apogee Santa Barbara 47P CCD camera. The larger main telescope is used for linear polarization observations with the Dipol-polarimeter (Piirola et al. 2005). The two telescopes are pointed to the same target and can be used simultaneously, if desired. 


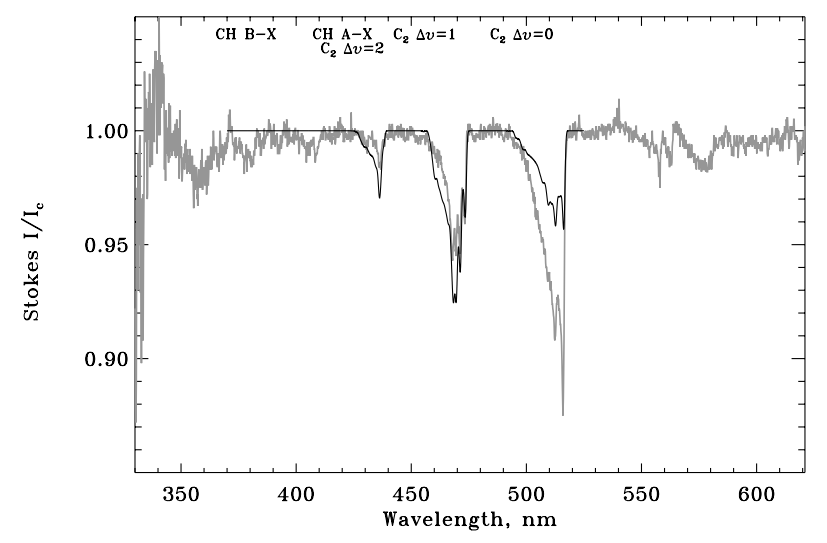

Fig. 1. Model fit from Berdyugina et al. (2005) applied to GJ893, one of the non-magnetic objects in our survey. Transition designations are given above the spectrum. The discrepancy between the depths of different Swan bands is obvious. The temperature of the model is $6000 \mathrm{~K}$.

Table 1. Observing log.

\begin{tabular}{lccc}
\hline \hline Object & Date & $N(B)$ & $N(R)$ \\
\hline EGGR78 & $2011-05-03$ & 26 & \\
& $2011-05-04$ & 13 & 14 \\
& $2011-05-05$ & 16 & \\
& $2011-05-09$ & 23 & \\
& $2011-05-10$ & 23 & \\
& $2011-05-12$ & 3 & \\
& $2011-05-13$ & 23 & \\
& $2011-05-15$ & 25 & \\
& $2011-05-16$ & 23 & \\
& $2011-05-17$ & 23 & \\
& $2011-05-20$ & 17 & \\
& $2011-05-22$ & 19 & \\
& $2011-05-23$ & 20 & \\
& $2011-05-24$ & 15 & \\
& $2011-05-29$ & 16 & \\
& $2011-05-30$ & 16 & \\
& $2011-05-31$ & 12 & \\
& $2011-06-02$ & 16 & \\
GJ1117 $2011-03-22$ & 7 & 8 \\
& $2011-03-23$ & 24 & \\
& $2011-03-24$ & 11 & \\
& $2011-03-25$ & 13 & \\
& $2011-03-28$ & 10 & \\
& $2011-03-29$ & 16 & \\
$2011-03-31$ & 27 & \\
\hline
\end{tabular}

We took data for GJ1117 during seven nights spread over ten nights and for EGGR78 during 17 nights spread over one month. We took exposures in both $B$ and $R$ bands on all nights. The dates of the observations and number of exposures for each night are listed in Table 1. Exposure times were $180 \mathrm{~s}$ for $B$-band and $100 \mathrm{~s}$ for $R$-band. We performed differential photometry comparing the objects to three comparison stars in the field of view. Since there is no information on the comparison stars apart from magnitudes in a few different bands (not $U B V R$ ), we also had to compare the comparison stars to each other to find out if any of them are variable. None of them were found to be variable within the errors. Figure 2 shows the lightcurves for EGGR78 and GJ1117 in $B$-band together with nightly averages compared to another star in the field of view. Errors of the individual measurements are from photon noise and the error on nightly averages are standard deviations of all measurements for the night in question. The magnitudes are arbitrary.
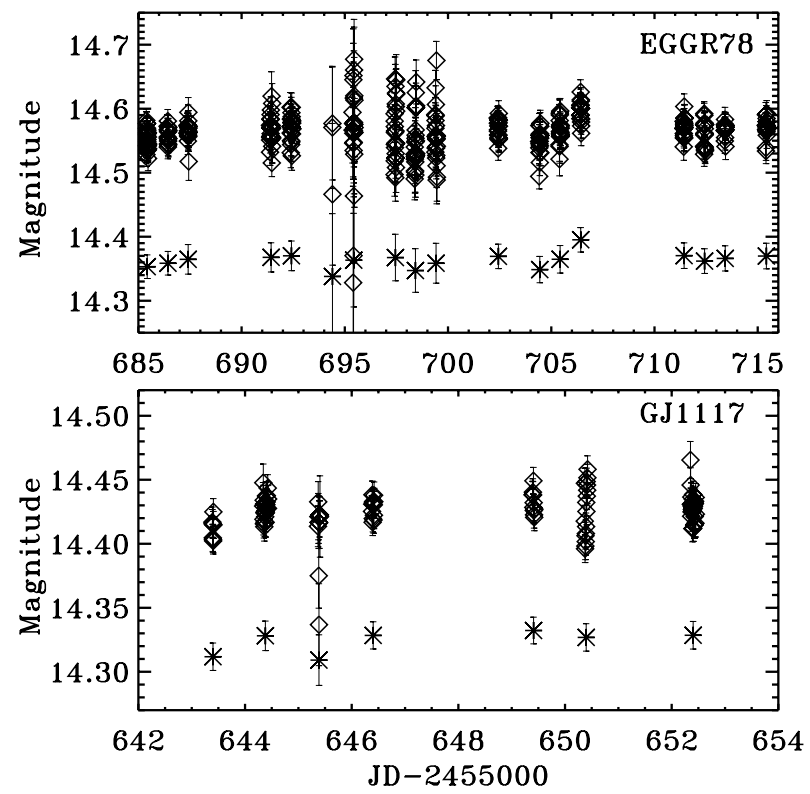

Fig. 2. Lightcurves for EGGR78 and GJ1117 together with nightly averages (displaced for clarity).

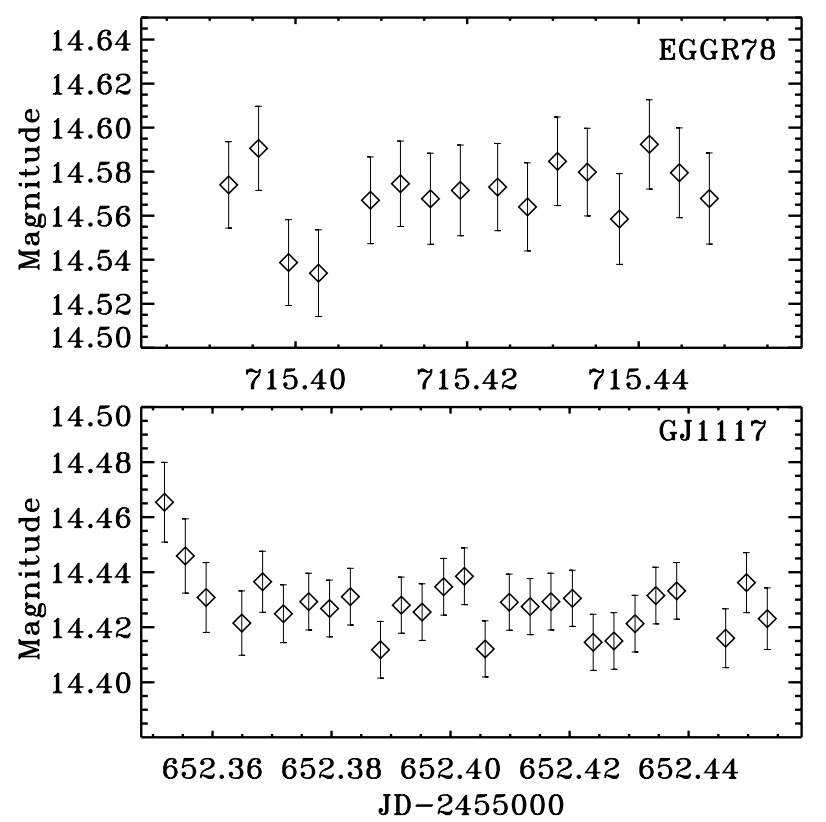

Fig. 3. Lightcurves for EGGR78 and GJ1117 from the last night of observations (June 2nd and March 31st, 2011, respectively).

The KVA telescopes are mostly used for optical monitoring of blazars in connection with the MAGIC Telescopes also located at ORM (See Albert et al. 2006, for example). The stability limit of the telescope is $15 \mathrm{mmag}$. As can be seen from Fig. 2, there is no real variability in EGGR78 or GJ1117 in $B$ or in $R$ band (not shown here).

Figure 3 shows as an example a lightcurve from the last night of observations for each star. No intra-night variability is evident in either object within errors.

The periodograms (Fig. 4) are also devoid of any signs of variability apart from the roughly 1 day period from the observing times. 
T. Vornanen and A. Berdyugin: Photometry of two DQ white dwarfs. Search for spots

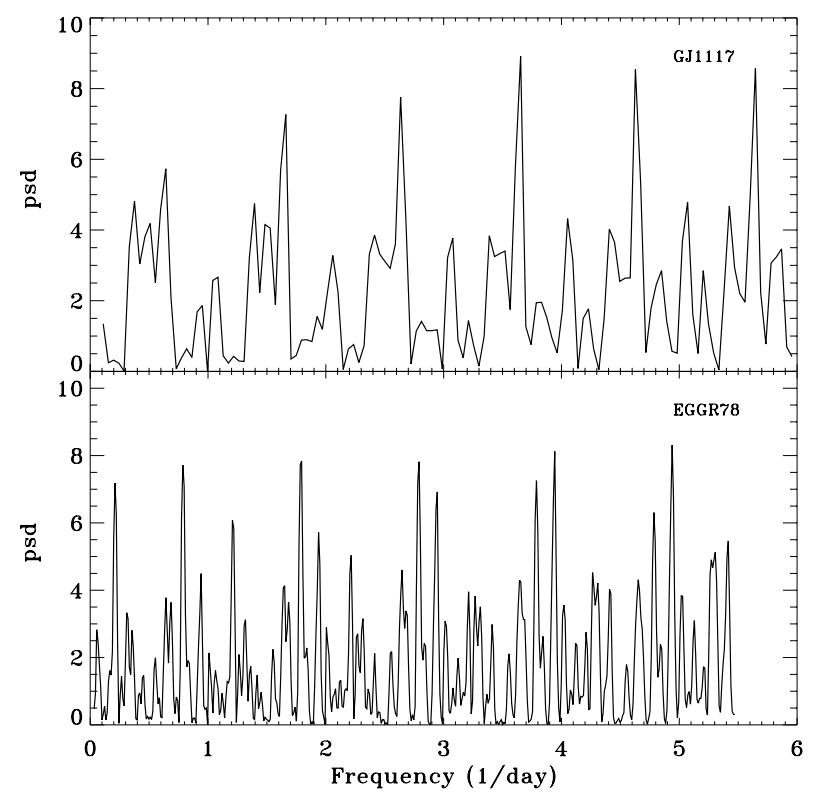

Fig. 4. Periodograms for GJ1117 (top) and EGGR78 (bottom) show no signs of periodic variability.

\section{Results}

Obtained lightcurves appear to be flat within the errors and neither inspection by eye, nor a Lomb-Scargle periodogram (Lomb 1976; Scargle 1982) can detect any kind of variability in our data set.

Since we did not find any signatures of variability in our photometry, we considered carefully to which kind of variations we are sensitive to and which we might be missing. We have two sources of uncertainty here. We might miss variability weaker than $15 \mathrm{mmag}$ because of the limiting accuracy of our small telescope, and the timings of our observations also limit our sensitivity to certain rotational periods.

To estimate the amplitude variations we could expect in the lightcurves, we used the model discussed earlier to calculate a lightcurve when a spot of higher $\mathrm{C}_{2}$ concentration and a temperature different from the atmospheric temperature crosses the visible hemisphere of the white dwarf. For simplicity, we assumed the spot to be a circular, flat disc. The angle between the normal of this disc and the line of sight is given by

$\cos \alpha=\cos (\beta) \sin (i) \cos (\phi)+\sin (\beta) \cos (i)$,

where $\beta$ is the spot latitude, $i$ is the inclination of the rotation axis from the line of sight, and $\phi$ is the rotational phase $(\phi=0$ towards the line of sight) (Brinkworth et al. 2005). Conveniently, this is also the size of the spot projected onto the plane of the sky. We also ignored limb-darkening effects for simplicity. The model produces normalised intensity spectra, therefore we had to transform our observed lightcurve from magnitudes to fluxes and then normalise them to the average value of the flux to obtain comparable results from observations and modelling.

We started by calculating the lightcurve from the spot/atmosphere combinations suggested by fitting the observed Stokes $I$ spectra of the two WDs. The temperatures in these two models are $2000 \mathrm{~K}$ for the atmosphere and $8000 \mathrm{~K}$ and $10000 \mathrm{~K}$ for the spots in EGGR78 and GJ1117, respectively, with spot sizes of $1 \%$ and $10 \%$. In both stars, modelling suggests that there is a low concentration of $\mathrm{C}_{2}$ in the atmosphere in general and the

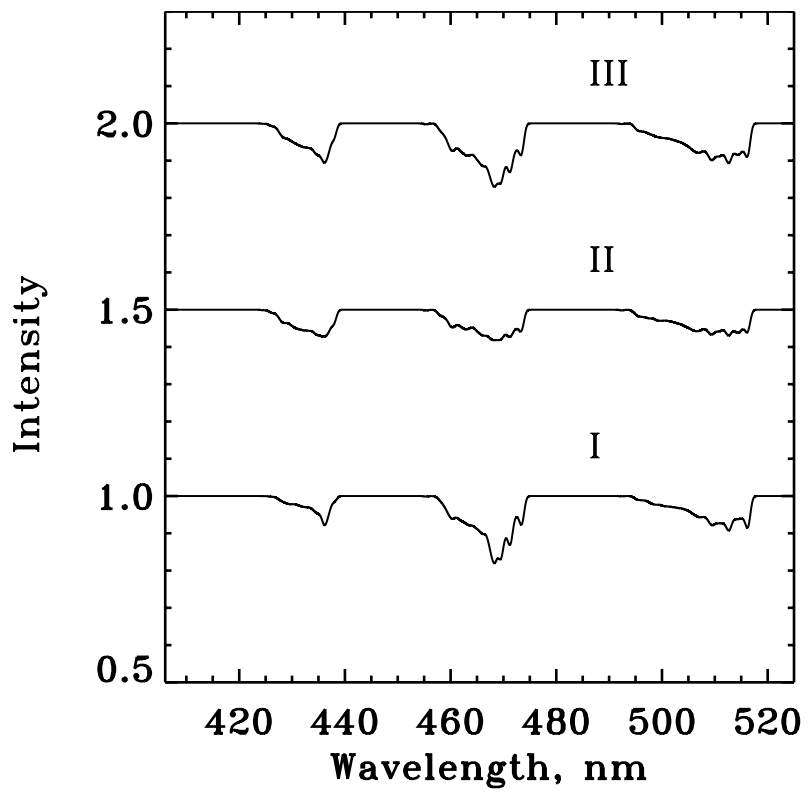

Fig. 5. Spot models: (I) homogenous atmosphere of $8000 \mathrm{~K}$, (II) spot of $8000 \mathrm{~K}$ in an atmosphere of $2000 \mathrm{~K}$, and (III) spot of $6000 \mathrm{~K}$ in an atmosphere of $8000 \mathrm{~K}$.
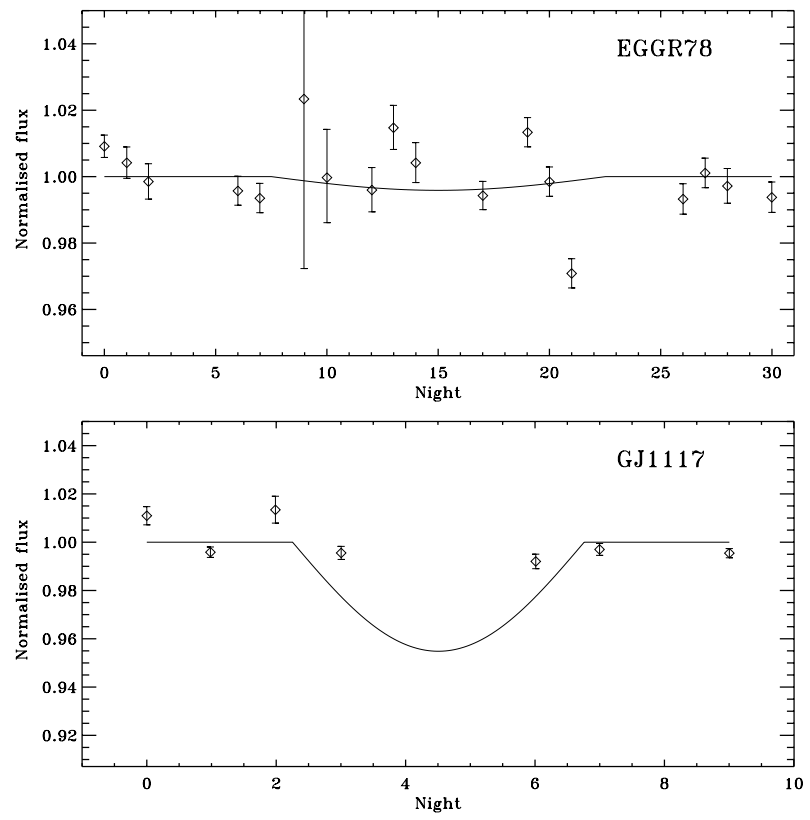

Fig. 6. Observed lightcurves (diamonds) of EGGR78 (top) and GJ1117 (bottom) together with model lightcurves (solid line).

spot has a much higher amount of $\mathrm{C}_{2}$. Figure 5 shows three examples of model spectra: (I) homogenous atmosphere at $8000 \mathrm{~K}$, (II) spot of $8000 \mathrm{~K}$ in an atmosphere of $2000 \mathrm{~K}$, and (III) a more realistic $6000 \mathrm{~K}$ spot in an atmosphere of $8000 \mathrm{~K}$. The differences between the spectra are very small, especially in cases I and III.

Since we do not know the rotational period of the white dwarfs, we used the time span of the photometric observations as the periods for illustration purposes. Results are shown in Fig. 6.

It is evident from Fig. 6 that the spot model that fits the spectrum of EGGR78 will not produce a sufficiently deep dip in the lightcurve for us to detect it. It would take a size of nearly $10 \%$ of the visible hemisphere for the spot to be detectable. This is 
because the $\mathrm{C}_{2}$ Swan bands on EGGR78 are very weak and the filter sensitivity makes the variability of the absorption bands even harder to detect, since the strongest band between 480 and $520 \mathrm{~nm}$ is not included in the $B$ filter. Variability on GJ1117 would clearly have been found if it were present.

The model lightcurves in Fig. 6 have the most ideal spot configuration for detection: namely, a spot on the equator of the star. When the spot is moved towards the pole, the drop in the lightcurve becomes shallower as the projected area of the spot, and its variation, becomes smaller. The same happens if the rotational axis is tilted from the plane of the sky towards the observer. This also changes the duration of the dip because the spot spends a different amount of time behind the star. Finally, when the spot latitude $\beta$ is higher than the inclination of the rotational axis $i$, the lightcurve assumes a true sine-form because the spot remains visible at all times but its projected size varies.

To estimate our chances of detecting a spot depending on the system geometry, we calculated a lightcurve for all angles of spot latitude $\left(-90^{\circ} \ldots 90^{\circ}\right)$ and inclination $\left(0^{\circ} \ldots 90^{\circ}\right)$ in one degree intervals. If the difference between maximum and minimum values of brightness on the lightcurve was larger than 0.03 , we counted it as detected. We also gave weights to the chances of detecting a spot for each latitude/inclination combination based on $\cos (\beta-i)$ to account for the fact that the points appear to be more densely distributed away from the line of sight towards the edges of the stellar disk. In this way we estimated the chances of missing a spot on both stars. Using a spot size of $10 \%$ we calculated that we would have had about a $40 \%$ chance of missing a spot in both our WDs using either model II or III.

There is, of course, the chance that we did not see variations in our photometric observations because the spot was behind the star during the observations. In this case, we might see some $\mathrm{C}_{2}$ that has spread out over the entire star, and missed the spot that is needed to model the spectra taken a year earlier. With the correct values of temperature and carbon abundance the spot can just alter the shape of the molecular absorption bands without changing their depths. The variations would be very difficult to pick up for the untrained eye by just looking at the spectra. Moreover, no one has tried monitoring these kinds of stars spectroscopically for a longer period to see if they are variable or not.

We should also consider the effect of a very long rotational period because our photometric observations as well as the spectropolarimetric observations might have been taken in the deepest part of the brightness drop. The normalisation level of the model lightcurve is determined by our spectropolarimetric observations, which were taken a year before the photometric observations, therefore a long rotational period could mean that all our observations have been performed during a minimum. For example, a 3000-day period would mean that both our spectropolarimetric and photometric observations could have been made in a low state.

\section{Conclusions}

We monitored two cool DQ WDs to search for photometric variability as a signature of a stellar spot. We did not find any such variability. The accuracy and timing of our observations do not allow us to detect very long rotational periods (of about a year or longer), very small spots, or some spot configurations. Previous studies of WDs have come to the conclusion that the rotation periods usually last from hours to decades (Charpinet et al. 2009, and references therein). Some magnetic WDs have indeed been found to be slow rotators with periods of about 100 years (Schmidt \& Norsworthy 1991; Berdyugin \& Piirola 1999). Since we did not detect any intra-night variability, the shorter end of the period distribution can be ruled out. Based on these arguments, we can assume that GJ1117 and EGGR78 still might have very long rotational periods.

Although we cannot rule out spots on these WDs completely, we consider the values given by our model for the spot sizes and temperatures to be very unrealistic. But if the spot model is not the solution to the discrepancy in intensity in the carbon molecular bands, what better hypotheses could be found? We think the answer may lie in the oscillator strengths of carbon molecules that are embedded in our model. These parameters determine the strengths of the individual absorption lines within the molecular bands. By modifying these values slightly, we hope to achieve consistent fits to all molecular bands without invoking a spot model. The current values were determined in laboratory conditions, but still contain significant error limits.

If modifying the oscillator strengths will not work, we have to go deeper into the physics of the problem and investigate in which way the structure, and therefore, the properties of a $\mathrm{C}_{2}$ molecule is different in a white dwarf from conditions in the laboratory where the molecule was studied. Or maybe our treatment of the WD atmosphere is incorrect and we have to consider its properties more thoroughly.

Acknowledgements. We would like to thank the referee for helpful comments. They improved this article immensely. T.V. would like to thank the Finnish Graduate School for Astronomy and Space Physics for financial support that has made this study possible. The authors would also like to express their gratitude to Kari Nilsson for his help in using the Diffphot photometry data reduction package that he has created, which we used to reduce the photometric data for this paper.

\section{References}

Albert, J., Aliu, E., Anderhub, H., et al. 2006, ApJ, 648, L105

Berdyugin, A. V., \& Piirola, V. 1999, A\&A, 352, 619

Berdyugina, S. V., Braun, P. A., Fluri, D. M., \& Solanki, S. K. 2005, A\&A, 444, 947

Berdyugina, S. V., Berdyugin, A. V., \& Piirola, V. 2007, Phys. Rev. Lett., 99, 091101

Bilíková, J., Chu, Y.-H., Su, K., Gruendl, R. A., \& Rauch, T. 2011, in Planetary Systems Beyond the Main Sequence: Proc. International Conference, eds. S. Schuh, H. Drechsel, \& U. Heber, AIP Conf. Ser., 1331, 215

Brinkworth, C. S., Marsh, T. R., Morales-Rueda, L., et al. 2005, MNRAS, 357, 333

Charpinet, S., Fontaine, G., \& Brassard, P. 2009, Nature, 461, 501

Dufour, P., Bergeron, P., \& Fontaine, G. 2005, ApJ, 627, 404

Lomb, N. R. 1976, Ap\&SS, 39, 447

Piirola, V., Berdyugin, A., Mikkola, S., \& Coyne, G. V. 2005, ApJ, 632, 576

Scargle, J. D. 1982, ApJ, 263, 835

Schmidt, G. D., \& Norsworthy, J. E. 1991, ApJ, 366, 270

Vornanen, T., Berdyugina, S. V., Berdyugin, A. V., \& Piirola, V. 2010, ApJ, 720, L52 